\title{
DỨBin
}

Technological University Dublin ARROW@TU Dublin

2019

\section{Some Transitivity-like Concepts in Abelian Groups}

\author{
Gabor Braun \\ Georgia Institute of Technology, gabor.braun@isye.gatech.edu \\ Brendan Goldsmith \\ Technological University Dublin, brendan.goldsmith@tudublin.ie \\ Ketao Gong \\ Hubei Engineering University, gketao@outlook.com
}

See next page for additional authors

Follow this and additional works at: https://arrow.tudublin.ie/scschmatart

Part of the Applied Mathematics Commons, and the Mathematics Commons

\section{Recommended Citation}

Braun, G., Goldsmith, B., Gong, K. \& Strungmann, L. (2019). Some transitivity-like concepts in Abelian groups. Journal of Algebra, 529(July), pp.114-123. doi:10.1016/j.jalgebra.2019.03.026

This Article is brought to you for free and open access by the School of Mathematics at ARROW@TU Dublin. It has been accepted for inclusion in Articles by an authorized administrator of ARROW@TU Dublin. For more information, please contact arrow.admin@tudublin.ie, aisling.coyne@tudublin.ie,gerard.connolly@tudublin.ie. 


\section{Authors}

Gabor Braun, Brendan Goldsmith, Ketao Gong, and Lutz Strungmann

This article is available at ARROW@TU Dublin: https://arrow.tudublin.ie/scschmatart/278 


\title{
Some Transitivity-like Concepts in Abelian Groups
}

\author{
Gábor Braun, Brendan Goldsmith, Ketao Gong, and Lutz Strüngmann \\ Abstract. The classical notions of transitivity and full transitivity in Abelian \\ $p$-groups have natural extensions to concepts called Krylov and weak transitivity. \\ The interconnections between these four types of transitivity are determined for \\ Abelian $p$-groups; there is a marked difference in the relationships when the prime \\ $p$ is equal to 2. In the final section the relationship between full and Krylov \\ transitivity is examined in the case of mixed Abelian groups which are $p$-local in the \\ sense that multiplication by an integer relatively prime to $p$ is an automorphism.
}

\section{Introduction}

The general notion of transitivity for an Abelian $p$-group was introduced by Kaplansky in [14]; subsequently this notion and the related notion of full transitivity appeared under the heading "Characteristic Submodules" as Section 18 of Kaplansky's famous "Little Red Book" [15]. Throughout, all groups will be additively written, reduced Abelian groups which will be local in the sense that only one arbitrary but fixed prime needs to be considered. Thus if the group is also torsion then it is a reduced $p$-group in the usual sense. We remark that it is not necessary to restrict our considerations to reduced groups but the additional generality obtained is of rather limited interest - see the discussion in Section 18 of [15].

The concept of a height sequence or Ulm sequence plays a key role. Recall that the height with respect to a prime $p$ of an element $x$ in the group $G$ (written $h_{G}(x)$ ) is the ordinal $\alpha$ if $x \in p^{\alpha} G \backslash p^{\alpha+1} G$ with the usual convention that $h(0)=\infty$. The Ulm sequence of $x$ with respect to $G$ is the sequence of ordinals or symbols 
$\infty$ given by $U_{G}(x)=\left(h_{G}(x), h_{G}(p x), h_{G}\left(p^{2} x\right), \ldots\right)$; the collection of such sequences will be partially ordered pointwise. Then a $p$-group $G$ is said to be transitive (fully transitive) if for each pair of elements $x, y \in G$ with $U_{G}(x)=U_{G}(y)\left(U_{G}(x) \leq U_{G}(y)\right)$ there is an automorphism (endomorphism) $\phi$ of $G$ with $\phi(x)=y$. Despite the suggestion inherent in the choice of names, the concepts are independent: Corner [1] exhibited a $p$-group which is fully transitive but not transitive and another group which is transitive but not fully transitive; note that every transitive group which is not fully transitive is necessarily a 2-group, a fact first shown by Kaplansky [15, Theorem 26]. Despite Corner's result showing the independence of the notions, there is a connection between the concepts: Files and Goldsmith [6] showed that a $p$-group $G$ is fully transitive if, and only if, $G \oplus G$ is transitive. The classes of groups which are both transitive and fully transitive are extensive and include the class of separable $p$-groups as well as the class of totally projective $p$-groups - see, for example, $[\mathbf{1 1}, \mathbf{1 3}, \mathbf{1 5}]$.

There is another natural concept which arises and seems to have been first investigated by Krylov [16] in the context of torsion-free groups: we say that a $p$-group $G$ is Krylov transitive if, given any pair of elements $x, y$ with $U_{G}(x)=U_{G}(y)$, there is an endomorphism $\phi$ of $G$ with $\phi(x)=y$. Clearly both fully transitive and transitive groups are Krylov transitive. Goldsmith and Strüngmann observed in [10] that the notions of full transitivity and Krylov transitivity coincide for $p$-groups when $p \neq 2$; note that this result fails if $p=2$ since Corner's [2] example of a transitive but not fully transitive 2-group is clearly Krylov transitive. An obvious question arises see, for example [3, Question 2.2] for this and a related query - is it possible to find a Krylov transitive group which is neither transitive nor fully transitive? We shall give a positive answer to this in Section 2 below. Our solution will depend heavily on a theorem due to Corner [1, Theorem 6.1] which we quote below in a slightly simplified form which is sufficient for our purposes:

TheOrem 1.1. Let $H$ be a countable bounded p-group, and let $\Phi$ be a countable subring of $\operatorname{End}(H)$. Then $H$ may be embedded as the subgroup $p^{\omega} G$ of elements of 
infinite height in a p-group $G$ such that $\operatorname{End}(G) \uparrow H=\Phi$ and $\operatorname{Aut}(G) \uparrow H=U(\Phi)$, the group of units of $\Phi$.

A further very general notion of transitivity was introduced by Goldsmith and Strüngmann in [9]: an arbitrary, not necessarily torsion group $G$ is said to be weakly transitive if, given $x, y \in G$ and endomorphisms $\phi, \psi$ of $G$ with $\phi(x)=y, \psi(y)=x$, there is an automorphism $\theta$ of $G$ with $\theta(x)=y$. Notice in this last concept that although there is no explicit reference to Ulm sequences, for a $p$-local group the existence of such endomorphisms $\phi, \psi$ ensures that $U_{G}(x)=U_{G}(y)$. Weak transitivity gives a connection between full transitivity and transitivity in the sense that a fully transitive, weakly transitive $p$-group is necessarily transitive. (The converse is not, however, true as was shown in [10, Corollary 3.13].) For $p$-groups with $p \neq 2$, the converse does, in fact, hold [10, Corollary 3.5]. We shall show that the Krylov transitive group constructed below which is neither transitive nor fully transitive, also fails to be weakly transitive.

We shall make repeated use of an observation due to Corner [2] that the transitivity or full transitivity of a $p$-group is determined by the action of the full endomorphism ring on the first Ulm subgroup $p^{\omega} G$; similar results are also easily established for Krylov and weak transitivity - see, for example, [3]. Specifically, a group $G$ is *-transitive, where *-transitivity denotes any one of transitivity, full transitivity, Krylov transitivity or weak transitivity, if given any pair of elements $x, y \in p^{\omega} G$ satisfying the appropriate condition of either $U_{G}(x)=U_{G}(y), U_{G}(x) \leq U_{G}(y)$ or in the case of weak transitivity, there exist endomorphisms of $G$ mapping $x \mapsto y$ and $y \mapsto x$, there is an endomorphism or automorphism (as appropriate) mapping $x \mapsto y$.

In the final section we consider the above notions in the context of mixed groups $M$ which are $p$-local in the sense that multiplication by an integer relatively prime to $p$ is an automorphism of $M$; one could, of course, just as easily consider $M$ as a module over the ring of integers localized at the prime $p$. Indeed this section could be extended to encompass the situation where $M$ is a mixed module over a discrete valuation ring. This section makes extensive use of ideas introduced by Files 
$[4,5]$. Our principal result is that Krylov transitivity and full transitivity coincide for mixed $p$-local groups having the property that the maximal divisible subgroup of the quotient of $M$ by its torsion subgroup, $d(M / t(M))$ has rank at most 1 - see Theorem 3.1.

Finally, our notation is standard and follows Fuchs $[7,8]$; in particular mappings are written on the left and $t(M)$ shall denote the torsion subgroup of the group M. Any notions relating to Abelian groups that are needed may be found in these works of Fuchs or in [15].

\section{Krylov Transitivity}

In this section we will answer a question posed in $[\mathbf{3}]$ by showing that there is a Krylov transitive 2-group that is neither transitive nor fully transitive. Moreover, we also prove that the group is not weakly transitive. We start with the basic construction.

Let $K=\bigoplus_{\aleph_{0}}\left\langle c_{i}\right\rangle \oplus(\langle a\rangle \oplus\langle b\rangle)$ where each of the $c_{i}$ is of order $2, a$ is of order 4 and $b$ is of order 16. We use the notation $K_{1}=\bigoplus_{\aleph_{0}}\left\langle c_{i}\right\rangle, K_{2}=\langle a\rangle \oplus\langle b\rangle$. Observe firstly that if $\Psi$ is a unital subring of $\operatorname{End}\left(K_{1}\right)$ and $\Phi$ is a unital subring of $\operatorname{End}\left(K_{2}\right)$, then $\Delta=\left(\begin{array}{cc}\Psi & \operatorname{Hom}\left(K_{2}, K_{1}\right) \\ \operatorname{Hom}\left(K_{1}, K_{2}\right) & \Phi+2 \operatorname{End}\left(K_{2}\right)\end{array}\right)$ is a unital subring of $\operatorname{End}(K)$; this follows easily from the observation that if $\beta \in \operatorname{Hom}\left(K_{2}, K_{1}\right)$ and $\alpha \in \operatorname{Hom}\left(K_{1}, K_{2}\right)$ then $\beta \alpha=0$ and $\alpha \beta \in 2 \operatorname{End}\left(K_{2}\right)$.

Now we specify the subrings $\Psi$ and $\Phi$ : for $\Psi$ we choose the subring of $\operatorname{End}\left(K_{1}\right)$ constructed by Corner which acts in a non-transitive but fully transitive manner on $K_{1}$ - see Section 3 in $[\mathbf{2}]$. For $\Phi$ we choose the subring of $\operatorname{End}\left(K_{2}\right)$ generated by $\operatorname{Aut}\left(K_{2}\right)$.

An examination of the lattice of Ulm sequences of $K_{2}$ - see Figure 1 - shows that $2 a+4 b$ and $2 a-4 b$ are the only elements of $K_{2}$ with Ulm sequence $(1,3, \infty, \ldots)$ and so the subgroup generated by these elements is invariant under the action of $\Phi$; thus the cyclic group $H=\{0,2 a+4 b, 8 b, 2 a-4 b\}$ is invariant under $\Phi$. 


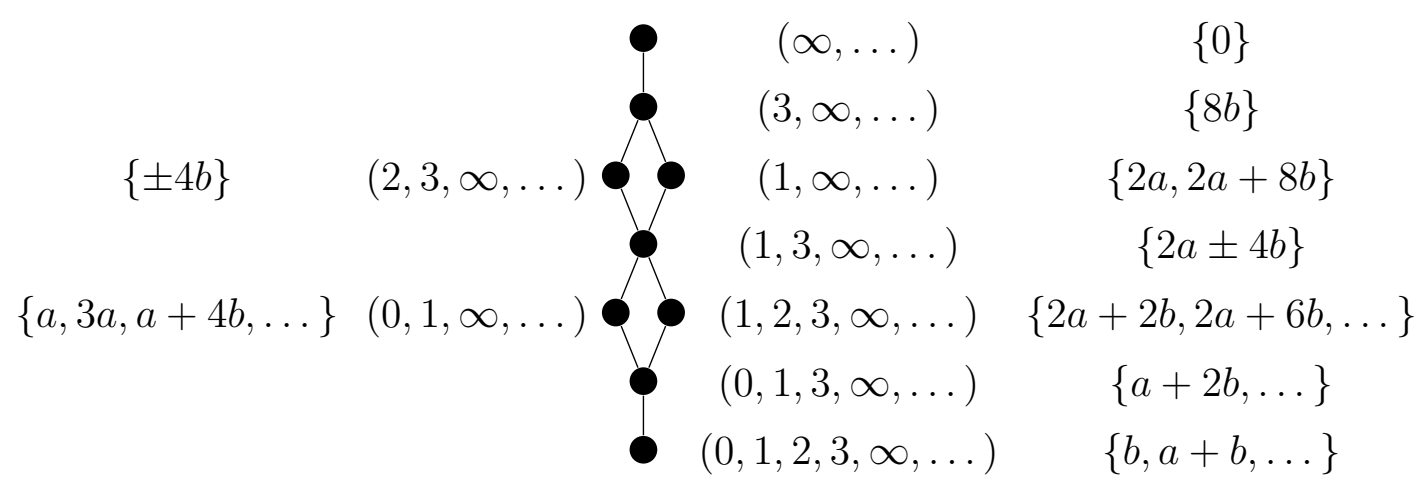

FIgURE 1. Lattice of Ulm sequences of $\mathbb{Z}(4) a \oplus \mathbb{Z}(16) b$

Furthermore, for any $\delta \in \operatorname{End}\left(K_{2}\right)$, we have that $2 \delta(H) \subseteq\{0,8 b\}$ and so $H$ is also invariant under the action of $\Phi+2 \operatorname{End}\left(K_{2}\right)$.

Applying Theorem 6.1 in $[\mathbf{1}]$ to the group $K$ and its unital subring $\Delta$, we obtain a group $G$ with $2^{\omega} G=K$ and $\operatorname{End}(G)\left\lceil 2^{\omega} G\right.$ acts as $\Delta$. To establish transitivity properties of $G$, it suffices to consider the action of $\Delta$ on the subgroup $K$.

Lemma 2.1. If the element $\left(\begin{array}{cc}\psi & \beta \\ \alpha & \phi+2 \delta\end{array}\right)$, where $\psi \in \Psi, \phi \in \Phi, \beta \in \operatorname{Hom}\left(K_{2}, K_{1}\right), \alpha \in$ $\operatorname{Hom}\left(K_{1}, K_{2}\right)$ and $\delta \in \operatorname{End}\left(K_{2}\right)$, is invertible, then $\psi$ is invertible.

Proof: Direct calculation shows that if $\left(\begin{array}{cc}\psi & \beta \\ \alpha & \phi+2 \delta\end{array}\right) \cdot\left(\begin{array}{cc}\psi_{1} & \beta_{1} \\ \alpha_{1} & \phi_{1}+2 \delta_{1}\end{array}\right)=I$, the identity matrix, then $\psi \cdot \psi_{1}=1_{K_{1}}$ as $\beta \alpha_{1}=0$. Reversing the order of multiplication gives $\psi_{1} \cdot \psi=1_{K_{1}}$ and so $\psi$ is invertible in $\Psi$.

Proposition 2.2. The group $G$ is neither transitive nor fully transitive.

Proof: Consider the elements $x=\left(\begin{array}{c}0 \\ 2 a+4 b\end{array}\right)$ and $y=\left(\begin{array}{c}0 \\ 2 a\end{array}\right)$ in $K$; they have Ulm sequences (in $G$ ) equal to $(\omega+1, \omega+3, \infty, \ldots)$ and $(\omega+1, \infty, \ldots)$ respectively. We claim that there is no endomorphism of $G$ mapping $x \mapsto y$; this will establish that $G$ is not fully transitive.

Suppose, for a contradiction, that there is an endomorphism of $G$ mapping $x \mapsto y$. Then there is an element $\left(\begin{array}{cc}\psi & \beta \\ \alpha & \phi+2 \delta\end{array}\right) \in \Delta$ with $\left(\begin{array}{cc}\psi & \beta \\ \alpha & \phi+2 \delta\end{array}\right)\left(\begin{array}{c}0 \\ 2 a+4 b\end{array}\right)=\left(\begin{array}{c}0 \\ 2 a\end{array}\right)$. 
This forces $(\phi+2 \delta)(2 a+4 b)=2 a$, which is impossible since, as noted above, $(\phi+2 \delta)(2 a+4 b) \in H=\{0,2 a+4 b, 2 a-4 b, 8 b\}$, while $2 a \notin H$. Thus the group $G$ is certainly not fully transitive.

To establish that $G$ is not transitive recall that Corner's construction of $\Psi$ leads to a non-transitive fully transitive group and so there are elements $x, y \in K_{1}$ with $U_{K_{1}}(x)=U_{K_{1}}(y)$ such that $\psi(x)=y$ for some $\psi \in \Psi$, but no unit of $\Psi$ can map $x \mapsto y$.

Now the elements $\left(\begin{array}{l}x \\ 0\end{array}\right),\left(\begin{array}{l}y \\ 0\end{array}\right)$ have equal Ulm sequences in $K$ since $U_{K_{1}}(x)=U_{K_{1}}(y)$. However, if there is a unit $\left(\begin{array}{cc}\gamma & \beta \\ \alpha & \phi+2 \delta\end{array}\right)$, with $\gamma \in \Psi$, which maps $\left(\begin{array}{l}x \\ 0\end{array}\right) \mapsto\left(\begin{array}{l}y \\ 0\end{array}\right)$, we must have $\gamma(x)=y$ and so $\gamma$ is not a unit of $\Psi$. This contradicts Lemma 2.1, so no such unit exists and consequently $G$ is not transitive.

Proposition 2.3. The group G is Krylov transitive.

To establish that $G$ is Krylov transitive, we consider pairs $\left(\begin{array}{l}x \\ y\end{array}\right),\left(\begin{array}{l}v \\ w\end{array}\right)$ of elements of $K$ having equal Ulm sequences. There are 4 possibilities:

1) $x=0, v=0$;

2) $x=0, v \neq 0$;

3) $x \neq 0, v=0$;

4) $x \neq 0, v \neq 0$.

Consider firstly the situations where $x \neq 0$. Then $U_{G}\left(\left(\begin{array}{c}2 x \\ 2 y\end{array}\right)\right)=U_{G}\left(\left(\begin{array}{c}2 v \\ 2 w\end{array}\right)\right)$ and then $U_{K_{2}}(2 y)=U_{K_{2}}(2 w)$. Since $K_{2}$ is transitive under $\Phi$, there exists $\phi \in \Phi$ with $\phi(2 y)=2 w$, so that $w-\phi(y) \in K_{2}[2]$. Hence $w-\phi(y)=z$ for some $z$ of order 2 in $K_{2}$. Now define $\alpha: K_{1} \rightarrow K_{2}$ by $\alpha(x)=z$ and choose $\psi \in \Psi$ such that $\psi(x)=v$ - both of these are possible: for the former it suffices to note that $z$ has order 2 and for the latter choice, the full transitivity of $K_{1}$ under $\Psi$ and the fact that $U_{K_{1}}(x) \leq U_{K_{1}}(v)$ suffice.

Then $\left(\begin{array}{cc}\psi & 0 \\ \alpha & \phi\end{array}\right)\left(\begin{array}{l}x \\ y\end{array}\right)=\left(\begin{array}{c}\psi(x) \\ \alpha(x)+\phi(y)\end{array}\right)=\left(\begin{array}{c}v \\ z+(w-z)\end{array}\right)=\left(\begin{array}{c}v \\ w\end{array}\right)$.

So in cases 3) and 4) there is an endomorphism mapping $\left(\begin{array}{l}x \\ y\end{array}\right) \mapsto\left(\begin{array}{l}v \\ w\end{array}\right)$.

Suppose now that $x=0=v$. Then $U_{K_{2}}(y)=U_{K_{2}}(w)$ and since $K_{2}$ is transitive under $\Phi$, there is a $\phi \in \Phi$ with $\phi(y)=w$. The diagonal matrix with entries $1, \phi$ is 
then an endomorphism (even an automorphism) in $\Delta$ mapping $\left(\begin{array}{l}0 \\ y\end{array}\right) \mapsto\left(\begin{array}{c}0 \\ w\end{array}\right)$, so Case 1) is also handled.

Consider now the remaining case, Case 2). Here we have $U\left(\left(\begin{array}{l}0 \\ y\end{array}\right)\right)=U\left(\left(\begin{array}{l}v \\ w\end{array}\right)\right)$ and $v \neq 0$.

It follows that $U\left(\left(\begin{array}{c}v \\ w\end{array}\right)\right)$ must be of the form $\left(0, \alpha_{1}, \alpha_{2}, \ldots\right)$, where $\alpha_{1}=h_{K_{2}}(2 w), \alpha_{2}=$ $h_{K_{2}}(4 w), \ldots$, while $U\left(\left(\begin{array}{l}0 \\ y\end{array}\right)\right)=U_{K_{2}}(y)$. Hence we conclude that $h_{K_{2}}(y)=0, h_{K_{2}}(2 y)=$ $\alpha_{1}=h_{K_{2}}(2 w), h_{K_{2}}(4 y)=\alpha_{2}=h_{K_{2}}(4 w), \ldots$ Examining the lattice of Ulm sequences of $K_{2}$, we see that $U(y)$ is either $(0,1, \infty, \ldots),(0,1,3, \infty, \ldots)$ or $(0,1,2,3, \infty, \ldots)$. In all three cases, $h_{K_{2}}(2 y)=1$, so $\alpha_{1}=h_{K_{2}}(2 w)=1$, forcing $h_{K_{2}}(w)=0$ also. Thus $U(y)=U(w)$ and so there is a $\phi \in \Phi$ with $\phi(y)=w$. Furthermore, since $h_{k_{2}}(y)=0$, the canonical projection $\eta: K_{2} \rightarrow K_{2} / 2 K_{2}$ gives $\eta(y) \neq 0$. But $\eta(y)$ then generates a summand of the vector space $K_{2} / 2 K_{2}$ and so there is a mapping $\theta: K_{2} / 2 K_{2} \rightarrow K_{1}$ with $\theta(\eta(y))=v$ and $\theta$ maps the complement of $\langle\eta(y)\rangle$ to 0 . The composition $\theta \eta$ is then a map from $K_{2} \rightarrow K_{1}$ with $\theta \eta(y)=v$. Then the map $\left(\begin{array}{cc}0 & \theta \eta \\ 0 & \phi\end{array}\right)$ which is an element of $\Delta$, takes $\left(\begin{array}{l}0 \\ y\end{array}\right)$ to $\left(\begin{array}{l}v \\ w\end{array}\right)$, so that in Case 2) we also have the required mapping. Thus $\Delta$ acts Krylov transitively on $K$ and, as noted in the Introduction, it follows from an easy extension of Corner's arguments in $[\mathbf{2}]$ that $G$ is Krylov transitive.

Proposition 2.4. If the p-group $G \oplus G$ is Krylov transitive, then $G$ is fully transitive.

Proof: Suppose for a contradiction that $G$ is not fully transitive. Then there must exist elements $x, y \in G$ with $U_{G}(x) \leq U_{G}(y)$ but no endomorphism $\eta$ of $G$ maps $x \mapsto y$.

Now consider the elements $\left(\begin{array}{l}x \\ x\end{array}\right)$ and $\left(\begin{array}{l}y \\ x\end{array}\right)$ in $G \oplus G$. Since $U_{G}(x) \leq U_{G}(y)$, it follows that $U_{G \oplus G}\left(\left(\begin{array}{c}x \\ x\end{array}\right)\right)=U_{G \oplus G}\left(\left(\begin{array}{c}y \\ x\end{array}\right)\right)$. However, if there is an endomorphism $\Theta \in \operatorname{End}(G \oplus$ $G)$ with $\Theta\left(\left(\begin{array}{l}x \\ x\end{array}\right)\right)=\left(\begin{array}{c}y \\ x\end{array}\right)$, then writing $\Theta=\left(\begin{array}{cc}\theta_{1} & \theta_{2} \\ \theta_{3} & \theta_{4}\end{array}\right)$, with each $\theta_{i} \in \operatorname{End}(G)$, we would have $\theta_{1}(x)+\theta_{2}(x)=y$. Making the standard identifications, this would establish the existence of an endomorphism $\theta=\theta_{1}+\theta_{2}$ of $G$ with $\theta(x)=y$-impossible. So $G \oplus G$ is Krylov transitive. 
THEOREM 2.5. There exists a non-transitive, non-fully transitive, non-weakly transitive group $G$ which is Krylov transitive. Furthermore, no direct power, $G^{(\alpha)}$ of $G$ is Krylov transitive for any cardinal $\alpha>1$.

Proof: For the first statement we need only show by Propositions 2.2 and 2.3 that $G$ is not weakly transitive. But this is immediate: a weakly transitive Krylov transitive group is necessarily transitive. The second statement follows since a summand of a Krylov transitive group is certainly Krylov transitive but we know from Proposition 2.4 that $G \oplus G$ is not Krylov transitive since $G$ is not fully transitive.

Note that the group $G$ in the above theorem gives a positive answer to Question 2.2 in $[3]$.

We conclude this section with clarifying the interconnection between the various notions of $*$-transitivity. A first example shows that there are weakly transitive p-groups which are not Krylov transitive.

LEMMA 2.6. Let $p$ be a prime. There exists a p-group $G$ which is weakly transitive but not Krylov transitive.

Proof: Let $H$ be an elementary $p$-group of rank 2 . Moreover, let $\Phi$ be the subring of the endomorphism ring $\operatorname{End}(H)$ generated by the identity. By Corner's realisation Theorem 1.1 there is a $p$-group $G$ such that the first Ulm subgroup of $G$ equals $H$ and $\operatorname{End}(G)$ acts on $H$ as $\Phi$. It is easily seen that $G$ is weakly transitive but not Krylov transitive.

We now display the interconnection of the $*$-transitivity notions for $p$-groups when $p=2$.

InTERCONNECTION 2.7. The following diagram shows the interconnection between the four notions of $*$-transitivity for $p$-groups in the case when $p=2$. 


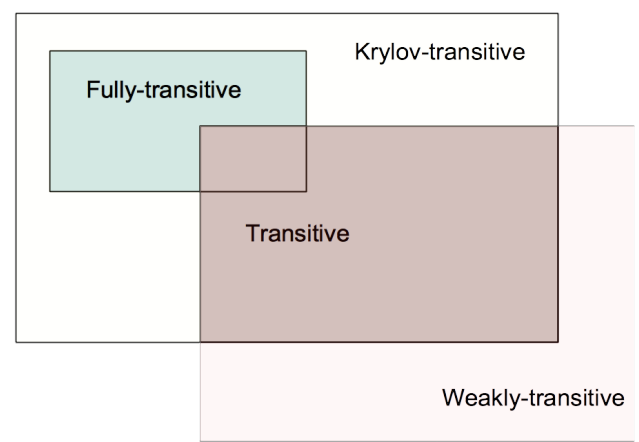

FIGURE 2. The interconnection of the four $*$-transitivity properties in the case when $p=2$.

By Corner's results we know that the two classes of transitive and fully transitive groups are different. Moreover, clearly full transitivity or transitivity induces Krylov transitivity and our main Theorem 2.5 shows that there is a Krylov transitive 2group that is neither transitive nor fully transitive. Finally, a transitive group is weakly transitive and if a group is weakly transitive and Krylov transitive, then it is transitive. A weakly transitive 2-group that is not Krylov transitive exists by Lemma 2.6.

We now pass to the case when $p \neq 2$.

INTERCONNECTION 2.8. The following diagram shows the interconnection between the four notions of $*$-transitivity for $p$-groups in the case when $p \neq 2$.

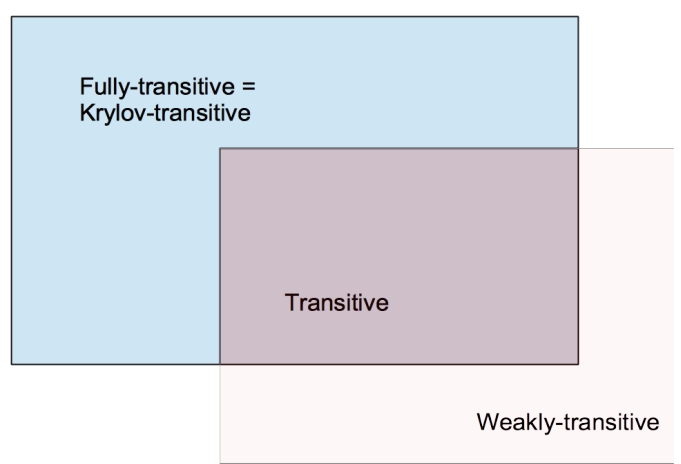

FIGURE 3. The interconnection of the four $*$-transitivity properties in the case when $p \neq 2$. 
By Kaplansky's result we know that transitivity implies full transitivity and by [10] we have that full transitivity is the same as Krylov transitivity. Finally, a transitive group is weakly transitive and if a group is weakly transitive and Krylov transitive, then it is transitive. A weakly transitive $p$-group for $p \neq 2$ that is not Krylov transitive is given in Lemma 2.6.

\section{Mixed groups}

We know from [10] that a $p$-group with $p \neq 2$ is Krylov transitive if, and only if, it is fully transitive. An obvious question is does a similar phenomenon occur for $p$-local groups when $p \neq 2$. We provide an affirmative partial answer. Let $t(M)$ denote the torsion subgroup of $M$. Recall that a group $M$ is $p$-local if multiplication by any integer relatively prime to $p$ is an automorphism of $M$; equivalently $M$ is a module over the ring $R$ of integers localized at $p$.

THEOREM 3.1. If $M$ is a p-local group $(p \neq 2)$ such that the divisible part $d(M / t(M))$ of the torsion-free quotient $M / t(M)$ has rank at most 1 , then $M$ is fully transitive if, and only if, it is Krylov transitive.

An obvious approach to this is to exploit Files's observation from [7] that a $p$-local group is fully transitive if, and only if, it is fully transitive over torsion and fully transitive mod torsion. Recall that a $p$-local group $M$ is fully transitive over torsion if for any $x \in M, y \in t(M)$, if $U_{M}(x) \leq U_{M}(y)$ then there exists an endomorphism of $M$ mapping $x \mapsto y$. The group $M$ is fully transitive mod torsion if for any $x, y \in M$ with $U_{M}(x) \leq U_{M}(y)$, there is an endomorphism $\phi$ of $M$ with $\phi(x)-y \in t(M)$. It is possible to modify Files's notions of transitivity and full transitivity over torsion and mod torsion to the Krylov concept. A $p$-local group $M$ is said to be Krylov transitive over torsion if for any $x \in M, y \in t(M)$, if $U_{M}(x)=U_{M}(y)$ then there exists an endomorphism of $M$ mapping $x \mapsto y$. The group $M$ is said to be Krylov transitive mod torsion if for any $x, y \in M$ with $U_{M}(x)=U_{M}(y)$, there is an endomorphism $\phi$ of $M$ with $\phi(x)-y \in t(M)$. 
REMARK 3.2. All the 'over torsion' types of transitivity imply that the torsion subgroup is transitive of that type, but the converse is not clear since endomorphisms of $t(M)$ do not necessarily extend to endomorphisms of $M$.

The proof of Theorem 3.1 is based on a series of arguments which are very similar to those in [5] and [12], proving for transitivity over torsion and mod torsion results similar to Theorem 3.1.

Proposition 3.3. If $M$ is a p-local group and the divisible part $d(M / t(M))$ of its torsion-free part $M / t(M)$ has rank at most 1 , then if $M$ is Krylov transitive mod torsion, it is fully transitive mod torsion.

Proof. Let $x, y \in M$ with $U_{M}(x) \leq U_{M}(y)$. We distinguish three cases depending on the places where the two sequences $U_{M}(x)$ and $U_{M}(y)$ coincide.

Case 1: $U_{M}(x)$ and $U_{M}(y)$ coincide on all but finitely many places. This means $U_{M}\left(p^{n} x\right)=U_{M}\left(p^{n} y\right)$ for some large enough $n$, hence by Krylov transitivity mod torsion, there is an endomorphism $\phi$ of $M$ such that $\phi\left(p^{n} x\right) \equiv p^{n} y \bmod t(M)$ and this clearly yields the desired $\phi(x) \equiv y \bmod t(M)$.

Case 2: $U_{M}(x)$ and $U_{M}(y)$ differ on all but finitely many places. Therefore $U_{M}\left(p^{n} x\right)<$ $U_{M}\left(p^{n} y\right)$ pointwise for large enough $n$, hence $U_{M}\left(p^{n} x\right)=U_{M}\left(p^{n}(x+y)\right)$. Now Krylov transitivity mod torsion yields an endomorphism $\phi$ with $\phi\left(p^{n} x\right) \equiv p^{n}(x+$ $y) \bmod t(M)$. Setting $\psi=\phi-1_{M} \in \operatorname{End}(M)$ we see that $\psi\left(p^{n} x\right) \equiv p^{n} y \bmod t(M)$ and this yields the desired $\psi(x) \equiv y \bmod t(M)$.

Case 3: $U_{M}(x)$ and $U_{M}(y)$ coincide on infinitely many places but also differ on infinitely many places.

It follows that each of $x$ and $y$ has infinitely many gaps in its height sequence, therefore by [12, Lemma 2.10], $x+t(M)$ and $y+t(M)$ both belong to $d(M / t(M))$. By hypothesis the rank of $d(M / t(M))$ is at most 1 , and therefore either $k x \equiv y$ $\bmod t(M)$ or $x \equiv p k y \bmod t(M)$ for some rational number $k$ whose denominator is not divisible by $p$. The latter case is impossible, as then $p^{t} x=p^{t+1} k y$ for some $t$, and hence $h_{M}\left(p^{t} x\right)>h_{M}\left(p^{t} y\right)$. If $h_{M}\left(p^{t} y\right)<\infty$, then this contradicts $U_{M}(x) \leq U_{M}(y)$. 
If $h_{M}\left(p^{t} y\right)=\infty$, then this contradicts the assumption that $U_{M}(y)$ has infinitely many gaps.

As a consequence, $k x \equiv y \bmod t(M)$, and hence the mappimg $\phi$ given by multiplication by $k$ is an endomorphism with $\phi(x) \equiv y \bmod t(M)$.

Our next result is an easy adaptation of results known in torsion groups.

LEMMA 3.4. Suppose that $x \in M$ and for all $y \in M[p]$ with $U_{M}(x) \leq U_{M}(y)$, there exists an endomorphism $\phi$ of $M$ with $\phi(x)=y$. Then for any $z \in t(M)$ with $U_{M}(x) \leq U_{M}(z)$, there is an endomorphism $\psi$ of $M$ with $\psi(x)=z$.

Proof. The proof is by induction on the order of $z$. The case $o(z)=1$, i.e., $z=0$ is obvious. So suppose the result is true for all elements of order $p^{n}$ in $M$ and that $o(z)=p^{n+1}$.

Now $U_{M}(x) \leq U_{M}(z)$ implies that $U_{M}(p x) \leq U_{M}(p z)$ and since $o(p z)=p^{n}$, we have, by induction, an endomorphism $\theta$ of $M$ with $\theta(p x)=p z$. Set $y=z-\theta(x)$, then clearly $y \in M[p]$ and $U_{M}(x) \leq U_{M}(y)$. Therefore by assumption, there is an endomorphism $\phi$ of $M$ with $\phi(x)=y$. Setting $\psi=\phi+\theta$ we get the desired result $\psi(x)=z$.

Proposition 3.5. If $M$ is a p-local group which is Krylov transitive, then if $p \neq 2$, $M$ is fully transitive over torsion.

Proof. Suppose that $x, y \in M$ with $U_{M}(x) \leq U_{M}(y)$ and $y \in t(M)$, we need to find an endomorphism $\phi$ of $M$ mapping $x \mapsto y$. It follows from Lemma 3.4 above that it suffices to consider the case where $y \in M[p]$.

The key observation is that since $h_{M}(x) \leq h_{M}(y)$, we have $h_{M}(x)=\min \left\{h_{M}(x+\right.$ $\left.y), h_{M}(x+2 y)\right\}$. In particular, $h_{M}(x+\varepsilon y)=h_{M}(x)$ with the choice of $\varepsilon=1$ or $\varepsilon=2$. Note that the condition $p \neq 2$ ensures that $\varepsilon$ is invertible even if $\varepsilon=2$.

By Krylov transitivity, there is an endomorphism $\phi$ of $M$ with $\phi(x)=\varepsilon y$. The mapping $\psi=\varepsilon^{-1} \phi$ then has the desired property that $\psi(x)=y$.

Proof of Theorem 3.1: 
If $M$ is fully transitive then it is certainly Krylov transitive (even if the rank of $d(M / t(M))$ is unrestricted).

Conversely suppose $M$ is Krylov transitive so that it is certainly Krylov transitive mod torsion. It follows from Proposition 3.3 that $M$ is then fully transitive mod torsion. Furthermore, it follows from Proposition 3.5 that $M$ is fully transitive over torsion.

The result now follows from Files's result [5] that a module is fully transitive if, and only if, it is fully transitive over torsion and fully transitive mod torsion.

\section{References}

[1] A.L.S. Corner, On endomorphism rings of primary Abelian groups II, Quart. J. Math. Oxford 27 (1976), 5-13.

[2] A.L.S. Corner, The independence of Kaplansky's notions of transitivity and full transitivity, Quart. J. Math. Oxford (2) 27 (1976), 15-20.

[3] P. Danchev and B. Goldsmith, On socle-regularity and some notions of transitivity for Abelian p-groups, J. Comm. Algebra (3) 3 (2011), 301-319.

[4] S. Files, On transitive mixed abelian groups, in "Abelian Group Theory: Proceedings of the International Conference at Colorado Springs", Lecture Notes in Pure and Appl. Math., Vol. 182, Dekker, New York (1996), 243-251

[5] S. Files, Transitivity and full transitivity for nontorsion modules, J. Algebra 197 (1997), 468478.

[6] S. Files and B. Goldsmith, Transitive and fully transitive groups, Proc. Am. Math. Soc. 126 (1998), 1605-1610.

[7] L. Fuchs, Infinite Abelian Groups, Vol. I and Vol. II, Academic Press 1970 and 1973.

[8] L. Fuchs, Abelian Groups, Springer International Publishing Switzerland 2015.

[9] B. Goldsmith and L. Strüngmann, Weakly transitive torsion-free abelian groups, Comm. Algebra 33 (2005), 1177 - 1191.

[10] B. Goldsmith and L. Strüngmann, Some transitivity results for torsion Abelian groups, Houston J. Math. 23 (2007), 941-957.

[11] P. Griffith, Transitive and fully transitive primary abelian groups, Pacific J. Math. 25 (1968), 249-254.

[12] G. Hennecke and L. Strüngmann, Transitivity and Full Transitivity for p-local Modules, Archiv der Mathematik 74 (2000), 321-329. 
[13] P. Hill, On transitive and fully transitive primary groups, Proc. Amer. Math. Soc. (2) 22 (1969), 414-417.

[14] I. Kaplansky, Some results on Abelian groups, Proc. Nat. Acad. Sci. 38 (1952), 538-540.

[15] I. Kaplansky, Infinite Abelian Groups, University of Michigan Press, Ann Arbor, 1954 and 1969.

[16] P. A. Krylov, Irreducible abelian groups and their endomorphism rings, Abelian groups and modules, Tomsk. Gos. Univ., Tomsk (1986), 73-100.

ISye, Georgia Institute of Technology, 755 Ferst Drive, NW Atlanta, GA 30332 , USA

E-mail address: gabor.braun@isye.gatech.edu

School of Mathematical Sciences, Dublin Institute of Technology, Aungier Street, DUBLin 2, IRELAND

E-mail address: brendan.goldsmith@dit.ie

School of Mathematics and Statistics, Hubei Engineering University, No. 272, JiaOtong Road, Xiaogan City, Hubei Province, 432000, P.R.China

E-mail address: gketao@outlook.com

Institute of Mathematical Biology, Faculty of Computer Sciences, Mannheim UniVersity of Applied Sciences, 68163 Mannheim, Germany

E-mail address: 1.struengmann@hs-mannheim.de 